\title{
A (SIMPLIFIED) BLUETOOTH MAXIMUM A POSTERIORI PROBABILITY (MAP) RECEIVER
}

\author{
R. Schiphorst, F.W. Hoeksema and C.H. Slump \\ Department of Electrical Engineering, Mathematics and Computer Science (EEMCS), \\ University of Twente, Enschede, The Netherlands, \\ Phone: (+31) (0)53489 2770, \\ email: [r.schiphorst, f.w.hoeksema, c.h.slump]@utwente.nl
}

\begin{abstract}
In our software-defined radio project we aim at combining two standards: Bluetooth and HiperLAN/2. The HiperLAN/2 receiver requires the most computation power in comparison with Bluetooth. We choose to use this computational power also for Bluetooth and look for more advanced demodulation algorithms such as a Maximum A posteriori Probability (MAP) receiver. This paper discusses a simplified MAP receiver for Bluetooth GFSK signals. The Laurent decomposition provides an orthogonal vector space for the MAP receiver. As the first Laurent waveform contains the most energy we have used only this waveform for our (simplified) MAP receiver. This receiver requires a $\frac{E_{b}}{N_{0}}$ of about $11 \mathrm{~dB}$ for a BER of $10^{-3}$, required by the Bluetooth standard. This value is about $6 \mathrm{~dB}$ better than single bit demodulators. This performance will only be met if the receiver has exact knowledge of the modulation index.
\end{abstract}

\section{INTRODUCTION}

Since the introduction of second generation mobile communication systems such as GSM, the mobile communication business has become a major business. Nowadays also other types of wireless communication such as wireless LAN or cordless telephone become popular. Furthermore there are not only different types of wireless communications but there is also an excess of standards for each type of wireless communication. For example for wireless LAN standards, the following standards exists: IEEE 802.11b, IEEE 802.11a, HiperLAN/1, HiperLAN/2, IEEE 802.11g, HomeRF etc. [1], [2].

In digital communication the trend is, due to Moore's law, that more functionality of the radio transceiver is implemented digital, because the analog part of the transceiver remains the same in every fabrication technology whereas the digital part is scaled down. So the transceiver is more and more digitized. It is for these two reasons, the digitalization of the transceiver and the abundance of standards which enables software (defined) radio.

\subsection{Outline}

The outline of this paper is as follows. First an introduction will be given on software (defined) radio and the software defined radio (SDR) project at the University of Twente. Then we discuss a Maximum A posteriori Probability (MAP) receiver which has two requirements; an orthogonal vector space and an efficient search algorithm. The orthogonal vector space is provided by the Laurent decomposition which will be discussed first. Then, the MAP receiver and its search algorithm will be described. Finally BER versus $E_{b} / N_{0}$ plots are shown and compared with a single bit detector and conclusions are drawn.

\subsection{Software radio}

The abundance of digital communication standards in not only disadvantageous for consumers but also for manufacturers because they have to develop a new product for each standard. It is for that reason that the software-radio concept is emerging as a potential pragmatic solution: a software implementation of the user terminal able to dynamically adapt to the radio environment in which the terminal is located [3]. For manufacturers this could result in shorter development time, cheaper production due to higher volumes. Furthermore SDR has advantages for consumers because it enables only software updates for new functionality without new hardware.

Because of the analog nature of the air interface, a software radio will always have an analog front end. In an ideal software radio, the analog-to-digital converter (ADC) and the digital-to-analog converter (DAC) are positioned directly after the antenna. Such an implementation is not feasible due to the power that such device would consume and other physical limitations [4][5]. It is therefore a challenge to design a system that preserves most properties of the ideal software radio while being realizable with current-day tech- 
nology. Such a system is called a software-defined radio (SDR).

There exists different points of views on software (defined) radio:

- The first line of thinking is to make current radio systems flexible in order to be able to correct design flaws as current designs become more complex e.g. a patchable radio. So a manufacturer can patch its products afterwards.

- The second point of view is to add flexibility and reconfigurability to radio hardware platforms to enable multi-standard receivers. This can be seen as a realization of the software defined radio concept.

- The third line of thinking is to implement radio algorithms using a general purpose processor (GPP). This can be seen as a realization of the software radio concept.

- The fourth point of view is to implement radio systems which dynamically adapt to the radio environment, communication needs and available resources. This can be seen as the realization of an adaptive radio.

\subsection{The Bluetooth HiperLAN/2 SDR receiver project}

In our SDR project [6] we aim at combining two different types of standards, Bluetooth [1] and HiperLAN/2 [2] on one common flexible hardware platform whereas our focus is on the physical layer of the receiver: from antenna output to raw bits. The research is carried out by two chairs of the University of Twente: the IC-Design group which focusses on the analog part and the chair Signals and Systems on the digital part.

Table 1 shows some characteristics of the physical layer of both standards. HiperLAN/2 is a high-speed Wireless LAN (WLAN) standard using Orthogonal Frequency Division Multiplexing (OFDM). Its physical layer is very similar to the 802.11a standard. Bluetooth on the other hand is a low cost, low speed standard, designed for replacing fixed cables. Bluetooth uses Gaussian Frequency Shift Keying (GFSK) which is also used by other standards such as IEEE 802.11b and DECT.

For our project we follow the second (and third line) of thinking of SDR: the HiperLAN/2 hardware is that complex to the Bluetooth hardware that the Bluetooth receiver may be added to the HiperLAN $/ 2$ at limited costs. In order to gain knowledge about Bluetooth and HiperLAN/ 2 receivers we have built a test-bed with two separate receivers [7]. The functional architecture is depicted in figure 1.

\begin{tabular}{|c|c|c|}
\hline parameter & Bluetooth & HiperLAN/2 \\
\hline $\begin{array}{c}\text { band } \\
\text { ch. spacing } \\
\text { modulation }\end{array}$ & $2.4-2.48 \mathrm{GHz}$ & $5.15-5.725 \mathrm{GHz}$ \\
& $1 \mathrm{MHz}$ & $20 \mathrm{MHz}$ \\
GFSK & OFDM + BPSK/ \\
(no FEC) & $1 \mathrm{Mbit} / \mathrm{s}$ & $12-72 \mathrm{Mbit} / \mathrm{s}$ \\
\hline
\end{tabular}

Table 1. Some physical layer characteristics of Bluetooth and HiperLAN/2

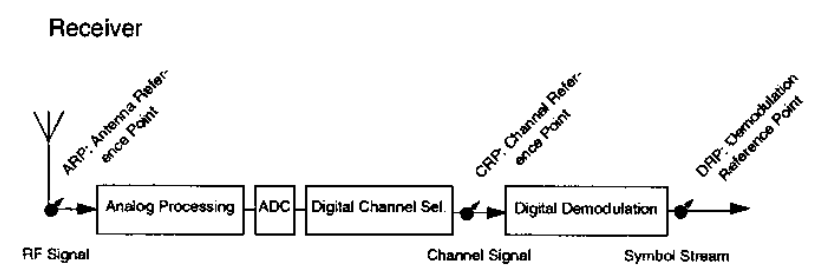

Fig. 1. Functional architecture of the SDR test bed

\section{LAURENT DECOMPOSITION}

The function of the channel-selection function (see figure 1) is to select one channel and to remove all others. Optimal demodulation is provided by a so-called Maximum A posteriori Probability (MAP) receiver. This receiver requires an orthogonal vector space which is given by the Laurent decomposition. This Laurent decomposition describes the GFSK signal by a sum of linear, orthogonal, Pulse AmplitudeModulated (PAM) waveforms.

\subsection{CPM signals}

Bluetooth uses Gaussian Frequency Shift Keying (GFSK) which belongs to the class of Continuous Phase Modulation (CPM) signals. A complex envelope of a CPM signal can be written as follows [8]:

$$
\tilde{s}(t, \alpha)=e^{j \psi(t, \alpha)}
$$

with

$$
\psi(t, \boldsymbol{\alpha})=h \pi \sum_{n} \alpha_{n} q(t-n T)
$$

In the equation above, $\mathrm{h}$ is the modulation index; $\alpha$, the symbol sequence belonging to the transmitted binary symbols $\left(\alpha_{n}=\{-1,1\}\right)$ and $q(t)$ is denoted as the phase response. For frequency modulation the relation between the phase and frequency response $g(t)$ is given by:

$$
q(t)=\int_{-\infty}^{t} g(\tau) \mathrm{d} \tau
$$

The phase response $\mathrm{q}(\mathrm{t})$ has the following properties:

$$
q(t)=0 \quad t \leq 0
$$




$$
q(t)=1 \quad t \geq L T
$$

with $\mathrm{T}$ is the bit duration and $\mathrm{L}$ is an integer value, which indicates the duration of the phase transition.

\subsection{Laurent decomposition}

In [9] it has been shown that equation 1 can be written as a sum of PAM waveforms (this is also deduced in the appendix of [10]):

$$
\tilde{s}(t, \alpha)=\sum_{k=0}^{Q-1} \sum_{n} b_{k, n} c_{k}(t-n T)
$$

with $Q=2^{L-1}$

The so-called pseudo symbols $b_{k, n}$ are given in equation 7 and the PAM waveform $c_{k}(t)$ in equation 9.

$$
b_{k, n}=\exp \left\{j h \pi\left[\left(\sum_{m=-\infty}^{n} \alpha_{m}\right)-\left(\sum_{i=0}^{L-1} \alpha_{n-i} \beta_{k, i}\right)\right]\right\}
$$

with $\alpha_{m}$ the $m^{\text {th }}$ data bit and $\beta_{k, i}$ is the $i^{\text {th }}$ bit of the so-called radix-2 representation of $k$ (, so $\beta_{k, i}$ has a value 0 or 1):

$$
k=\sum_{i=1}^{L-1} 2^{i-1} \beta_{k, i} \quad 1 \leq k \leq Q-1
$$

$c_{k}(t)$ is a product of functions $u(t)$ :

$c_{k}(t)=u(t) \prod_{i=1}^{L-1} u\left(t+i T+L T \beta_{k, i}\right) \quad 1 \leq k \leq Q-1$

where the function $u(t)$ is defined as follows:

$$
\begin{array}{ll}
u(t)=\sin (h \pi q(t)) / \sin (h \pi) & 0 \leq t \leq L T \\
u(t)=u(2 L T-t) & L T \leq t \leq 2 L T \\
u(t)=0 & \text { elsewhere }
\end{array}
$$

From equation 10 it can be seen that the function $u(t)$ is symmetric around $t=L T$ and has a duration of $2 L T$.

In many cases the signal power is concentrated in the first pulse, $c_{0}$. So, the CPM signal can be approximated by using only this pulse (which simplifies the construction of the MAP receiver):

$$
\hat{\tilde{s}}(t, \alpha) \approx \sum_{n} b_{0, n} c_{0}(t-n T)
$$

\subsection{Laurent decomposition of Bluetooth GFSK}

This section derives the Laurent decomposition for Bluetooth GFSK modulation. The frequency response function $g(t)$ is equal to the response of "one bit" to a Gaussian filter:

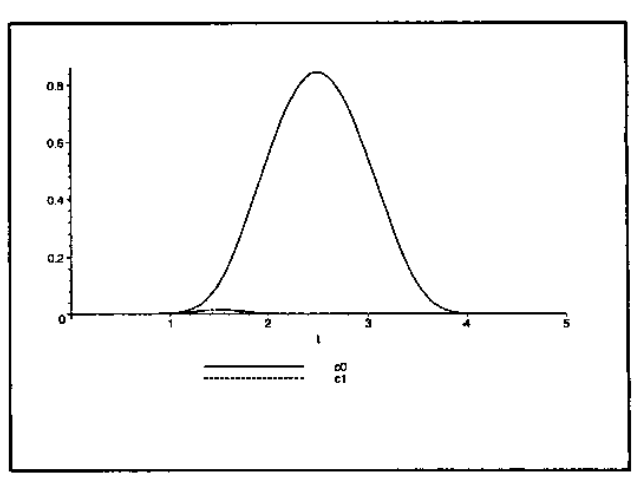

(a) $c_{0}$ and $c_{1}$

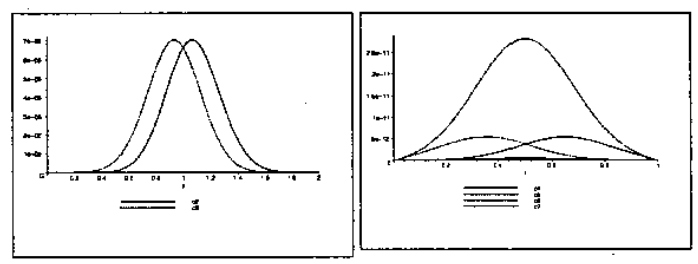

(b) $c_{2}$ and $c_{3}$

(c) $c_{4}, c_{5}, c_{6}$ and $c_{7}$

Fig. 2. Signal components of GFSK with $B T=0.5$ and $L=4$

$$
f(t)=\frac{1}{2}\left(\operatorname{erf}\left(\alpha\left(\frac{t}{T}-\frac{1}{2}\right)\right)+\operatorname{erf}\left(\alpha\left(\frac{t}{T}-\frac{1}{2}\right)\right)\right)
$$

with:

$$
\alpha=\pi B T \frac{\sqrt{2}}{\ln (2)}
$$

and with the $\mathrm{BT}$ product, $B T=0.5$

The modulation index can vary between 0.28 and 0.35 according [1]. As the smallest modulation index gives the worst performance (at the same noise power), we used this modulation index for the Laurent decomposition. The resulting PAM waveforms (with $L=4$ ) are shown in figure 2 for a modulation index $h$ of 0.28 . From the figure, it can be seen that the first waveform $c_{0}$ is the most important pulse.

Figure 3 shows the phase of an example GFSK signal and its Laurent approximation. Note that the approximation, using only the first Laurent term, and neglecting transients effects, already equals the original GFSK signal. Zooming in (figure 4) reveals that there are some small differences. 


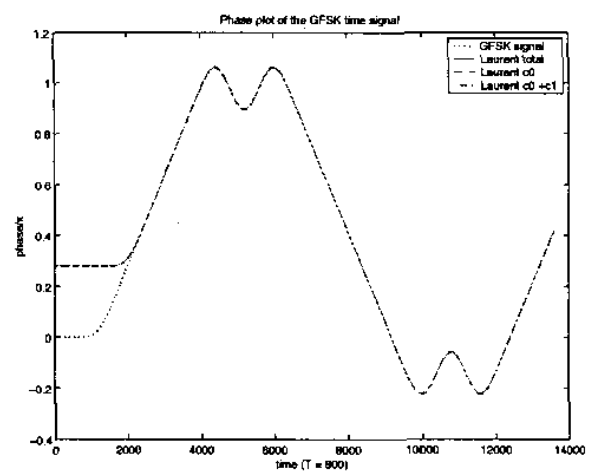

Fig. 3. Phase plot of an example GFSK signal and its Laurent decompositions

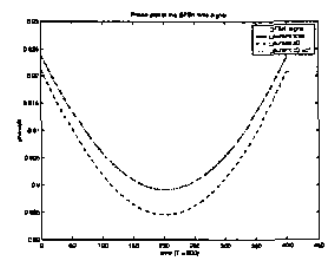

Fig. 4. A zoomed-in version of figure 3

\section{A MAXIMUM A POSTERIORI PROBABILITY (MAP) RECEIVER}

In the previous section an orthogonal vector space has been derived that can be used in a MAP receiver. Only the first Laurent waveform is important, other waveforms can be neglected, especially if the noise power is high. Following the Gram-Schmidt procedure we have to normalize and mirror the first Laurent waveform:

$$
H_{0}(i)=\frac{c o(-t)}{\left\|c_{0}(t)\right\|}
$$

The MAP receiver is shown in figure 5. Recall that the Laurent approximation of the GFSK Bluetooth signal is (eq. 11):

$$
\tilde{r}(t, \alpha) \approx \sum_{n} b_{0, n} c_{0}(t-n T)
$$

So, the filter $H_{0}(t)$ is a matched filter for the first Laurent waveform. Therefore the output of the filter is an (optimal) estimation of $b_{0, n}$. This estimation has an optimal $\frac{E_{b}}{N_{0}}$ but suffer also from Inter-Symbol Interference (ISI). So an

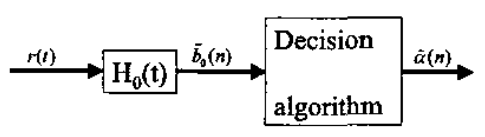

Fig. 5. MAP receiver

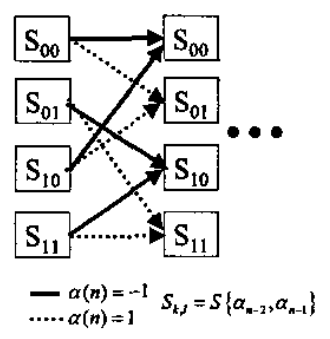

Fig. 6. Viterbi algorithm

efficient search algorithm will be needed which determines the optimal path through the trellis diagram. For our MAP receiver we used the Viterbi algorithm.

As the Gaussian filter has an filter length of about 3 bit times, a Viterbi algorithm with maximal 2 state variables should be sufficient; $\left(\alpha_{n-2}\right.$ and $\left.\alpha_{n-1}\right)$. The states and their branches to the next states are shown in figure 6 .

\subsubsection{Steps in the Viterbi algorithm}

Every sample the Viterbi algorithm must:

- calculate all 8 the branch metrics (, see equation 16)

- as two paths enter each state, save only the path with the highest state, the other can be discarded.

- determine the state with the highest value and then decide the value of $\alpha_{n-2}$, and update the state variables $\alpha_{n-2}, \alpha_{n-1}$ and $b_{n-3}$

The Viterbi algorithm can be initialized by setting the first sample to $b_{n-3}$ and setting all states to zero. Then the algorithm starts at the $4^{\text {th }}$ sample for decoding the first bit.

The branch metric is defined as follows:

$$
B M=b_{0, n-3} \exp \left(j h \pi \alpha_{n-2}+j h \pi \alpha_{n-1}+j h \pi \alpha_{n}\right) \tilde{b}_{0, n}^{*}
$$

where $\tilde{b}_{0, n}$ is the output of filter $H_{0}(t)$.

\subsection{Results}

In Figure 7 the performance of the MAP receiver is depicted for several modulation indexes and for several Viterbi algorithms. For each $\frac{E_{b}}{N_{0}}$ value 500000 bits have been simulated. Furthermore we used 80 samples per symbol in our simulation model. After the $H_{0}$ filter, the sample stream was decimated with a factor 10 , so 8 samples per symbol were used for synchronization and bit detection. A $\frac{E_{b}}{N_{0}}$ (for the smallest modulation index) of about $11 \mathrm{~dB}$ is required for a BER of $10^{-3}$ (required by the Bluetooth standard). 


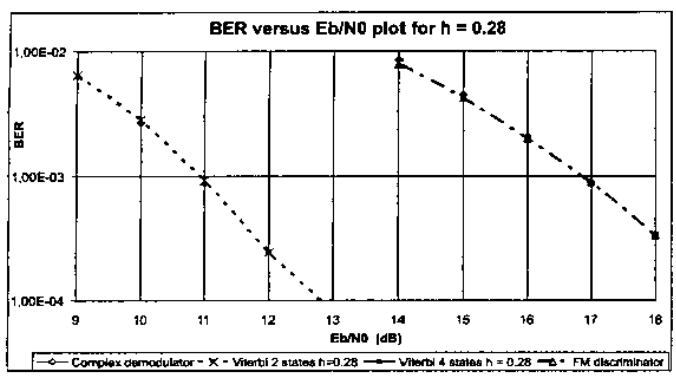

Fig. 7. BER versus $E_{b} / N_{0}$ plot

\section{CONCLUSIONS AND RECOMMENDATIONS}

In this paper we have derived a (simplified) MAP receiver for Bluetooth signals. This receiver requires (for the smallest modulation index) a $\frac{E_{b}}{N_{0}}$ of about $11 \mathrm{~dB}$ for a BER of $10^{-3}$ (required by the Bluetooth standard). From figure 7 it can seen, that the Viterbi algorithm with 2 states has only a small performance degradation compared with the 4-state algorithm.

Other demodulators, such as single bit detectors have worse performance. In our simulation model we have also implemented an FM discriminator which requires about a $\frac{E_{b}}{N_{0}}$ of $17 \mathrm{~dB}[11]$. The value equals the one found in literature [12]. We have seen in simulations that a direct conversion demodulator [13], requires the same $\frac{E_{b}}{N_{0}}$. So the performance gain is $6 \mathrm{~dB}$.

A MAP receiver needs exact knowledge of the modulation index because the performance is very sensitive for the modulation index error. A possible solution to this problem is to estimate the modulation index from the first, known, part of the Bluetooth packet, the access code. Further research will have to verify this. Another solution is the use of adaptive search algorithms, such as an Decision Feedback Equalizer (DFE).

\section{Acknowledgment}

We thank our colleagues from the IC-Design group of the University of Twente for their work on the analog part. Moreover we thank Kiwi Smit of Ericsson Eurolab Netherlands B.V. for his comments. This research is supported by the PROGram for Research on Embedded Systems \& Software (PROGRESS) of the Dutch organization for Scientific Research NWO, the Dutch Ministry of Economic Affairs and the technology foundation STW.

\section{REFERENCES}

[1] Bluetooth SIG, "Specification of the bluetooth system - core," Technical Specification Version 1.1, Bluetooth
SIG, February 2001.

[2] ETSI, "Broadband radio access networks (bran); hiperlan type 2; physical (phy) layer," Technical Specification ETSI TS 101475 V1.2.2 (2001-02), ETSI, 650 Route des Lucioles - Sophia Antipolis, Valbonne - FRANCE, February 2001.

[3] J. Mitola, Software Radio Architecture: ObjectOriented Approaches to Wireless Systems Engineering, Wiley, 2000.

[4] R.H. Walden, "Performance trends for analog-todigital converters," IEEE Communications Magazine, pp. 96-101, February 1999.

[5] V.J. Arkesteijn, E.A.M. Klumperink, and B. Nauta, "An analogue front-end architecture for software defined radio," $13^{\text {th }}$ proRISC workshop, November 2002.

[6] "The bluetooth-hiperlan/2 sdr receiver project," http://nt5.el.utwente.nl/sar/.

[7] V.J. Arkesteijn, R. Schiphorst, F.W. Hoeksema, E.A.M. Klumperink, B. Nauta, and C.H. Slump, "A software-defined radio test-bed for wlan front ends," $3^{\text {nd }}$ PROGRESS workshop on Embedded Systems and Software, 2002.

[8] U. Mengali, "Decomposition of m-ary cpm signals into pam waveforms," IEEE transactions on information theory, vol. 41, no. 5, pp. 1265-1275, September 1995.

[9] P.A. Laurent, "Exact and appropiate construction of digital phase modulateds by superposition of amplitude modulated pulses (amp)," IEEE transactions on communications, vol. 34, no. 2, pp. 150-160, February 1986.

[10] G. Kawas Kaleh, "Simple coherent receivers for partial response continous phase modulation," IEEE Journal on Selected Areas in Communications, vol. 7, no. 9, pp. 1427-1436, December 1989.

[11] R. Schiphorst, F.W. Hoeksema, and C.H. Slump, "Bluetooth demodulation algorithms and their performance," Workshop on Software Radios (WSR2002), Karlsruhe (G), March 2002.

[12] A. Soltanian and R.E. Van Dyck, "Performance of the bluetooth system in fading dispersive channels and interference," Proceedings of IEEE Globecom, November 2001 .

[13] M.H.L. Kouwenhoven, High-Performance Frequency-Demodulation Systems, Ph.D. thesis, Delft University of Technology, 1998. 